


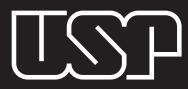

Universidade de São Paulo

Prof. Dr. João Grandino Rodas Reitor

Prof. Dr. Hélio Nogueira da Cruz Vice-reitor

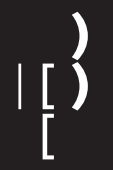

Instituto de Estudos Brasileiros

Prof. ${ }^{a}$ Dr. ${ }^{a}$ Maria Angela Faggin Pereira Leite Diretora

Prof. ${ }^{\mathrm{a}}$ Dr. ${ }^{\mathrm{a}}$ Marina de Mello e Souza Vice-diretora 


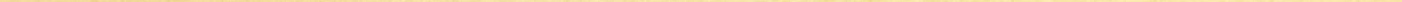




\section{Revista do Instituto de Estudos Brasileiros}

\section{ISSN 0020-3874}

\section{número 57, 2013 jul./dez.}

Comissão EDITORIAL

Denilson Lopes Silva (UFRJ) Rio de Janeiro, BR

Gustavo Alejandro Sorá (UNC) Córdoba, AR

Jaime Tadeu Oliva (IEB-USP) São Paulo, BR

Paulo Teixeira Iumatti (IEB-USP) São Paulo, BR

Pedro Meira Monteiro (Princeton U.) Princeton,

EUA

Randal Johnson (UCLA) Los Angeles, EUA

Walter Garcia (IEB-USP) São Paulo, BR

Editores Responsáveis

Jaime Tadeu Oliva (IEB-USP)

Walter Garcia (IEB-USP)

Editor Adjunto

Paulo Teixeira Iumatti (IEB-USP)

Dossié Mário de Andrade - organização

Walter Garcia (IEB-USP)

Colaboração

Alexandre de Freitas Barbosa (IEB-USP)

Assistentes editoriais

Fernanda Rodrigues Rossi

José Hermes Martins Pereira

Lia Marques (estagiária)

Equipe de apoio

Regina Mayumi Aga

Eduardo Henrique Gorobets Martins (estagiário)

Editoração eletrônica

Beatriz Garcia (estagiária)

Iris Fabrin Sototuka (estagiária)

Colaboraram neste número

Profa. Dra. Marisa Midori Deaecto

Inês de Araújo (trad. língua francesa)

Alícia Toffani (preparação de texto)

Marina Neofiti (revisão de texto)

Projeto gráfico

Homem de Melo \& Troia Design

Impressão:

\section{Distribuição:}

Editora 34

R. Hungria, 592

01455-00o São Paulo SP

(11) 38166777 vendas@editoraz4.com.br

Conselho Consultivo

Adrián Gorelik

Universidade Nacional de Quilmes, Bernal, AR

Barbara Weinstein

Universidade de Nova lorque, Nova lorque, EUA

Carlos Augusto Calil

Universidade de São Paulo, São Paulo, BR

Carlos Sandroni

Universidade Federal de Pernambuco, Recife, BR

Ettore Finazzi-Agrò

Universidade de Roma La Sapienza, Roma, IT

Fernanda Arêas Peixoto

Universidade de São Paulo, São Paulo, BR

Heloisa Maria Murgel Starling

Universidade Federal de Minas Gerais, Belo Horizonte, BR

João Cezar de Castro Rocha

Universidade Estadual do Rio de Janeiro, Rio de Janeiro, BR

Jorge Coli

Universidade Estadual de Campinas, Campinas, BR

Luiz Felipe de Alencastro

Universidade de Paris-Sorbonne, Paris, FR

Manuel Villaverde Cabral

Universidade de Lisboa, Lisboa, PT

Maria Cecilia França Lourenço

Universidade de São Paulo, São Paulo, BR

Maria Ligia Coelho Prado

Universidade de São Paulo, São Paulo, BR

Maria Lucia Bastos Kern

Pontificia Universidade Católica do Rio Grande do Sul,

Porto Alegre, BR

Peter Burke

Emmanuel College Cambridge, Cambridge, RU

Regina Zilberman

Universidade Federal do Rio Grande do Sul, Porto Alegre, BR

Ricardo Augusto Benzaquen de Araújo

Pontificia Universidade Católica do Rio/ Instituto Universi-

táriode Pesquisas do Rio de Janeiro, Rio de Janeiro, BR

Rodolfo Nogueira Coelho de Souza

Universidade de São Paulo, São Paulo, BR

Sergio Miceli

Universidade de São Paulo, São Paulo, BR

Walnice Nogueira Galvão

Universidade de São Paulo, São Paulo, BR

Instituto de Estudos Brasileiros

Edifício Brasiliana, Praça do Relógio Solar, 342

Cidade Universitária

05508-050, São Paulo - SP, Brasil

(11) 30911149

www.ieb.usp.br 
guenides Mario

4 cabo de ruseber a tina sugunda remena de cunta, qus tanatoínownojadsra,

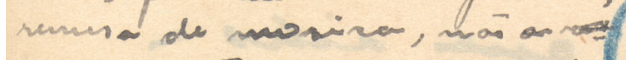
pare prate de minbor wind mo voce mi falo centas a primusect Lumides ario ando meto histe Zueri da toria que para min fob

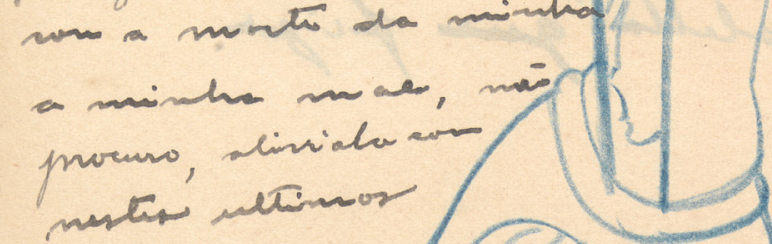
sei co mo seffagar esta min ho do - mer trabolto, qu ginstemanto

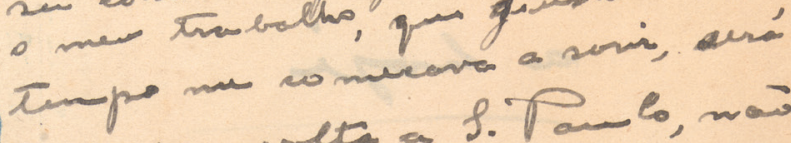

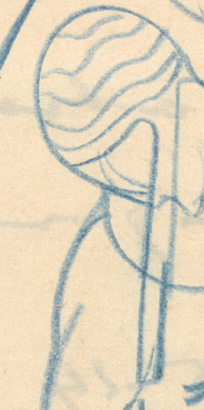
ninha wotta a f. Pan lo, now bue triota a ucestion a

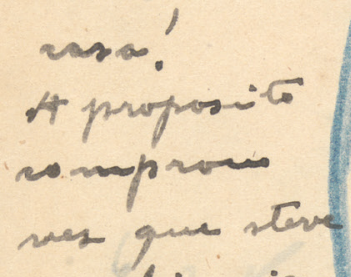
que ficonio foger antis ninka min, en minka men me perguntas se D. Glivi a miser bonber blla a well no men Atclie, me den ape

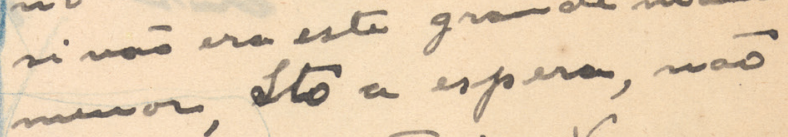

tives aimde de Ganniller, animä́rella a dimi our pos min servia na grande cinsa mete m pona mix

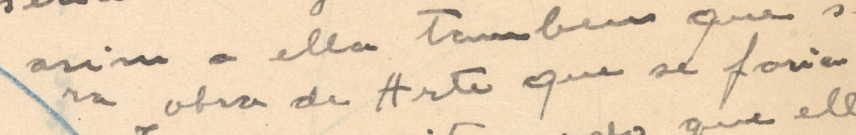
Gencho minito medo que elle mojetionar por algun, is idenion, Por favin mario no Bresil se anite poran on fogs de ence Topuc or ponba un i.emanto ra A respicito da maximo da joggo an volumer arpiacer, g

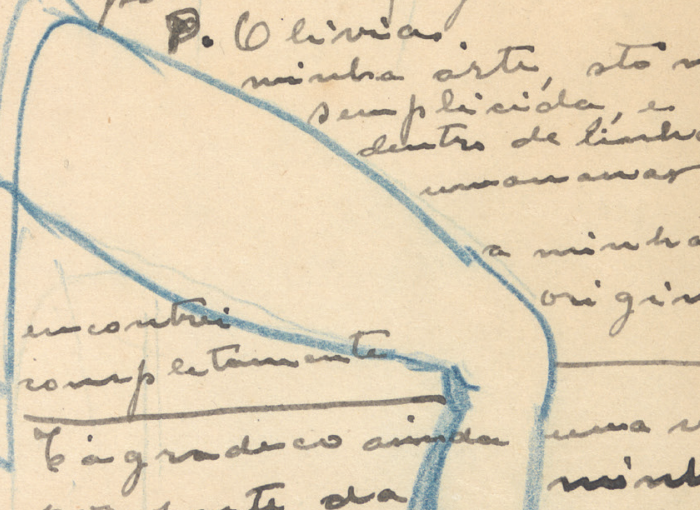

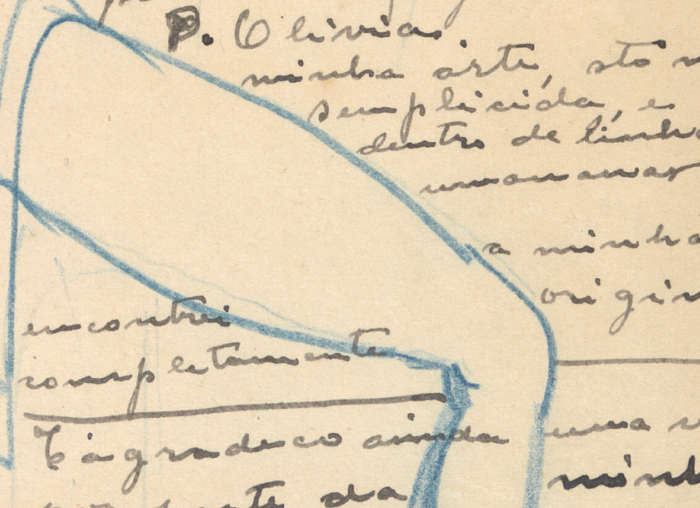
gracos a d De gien par perte da

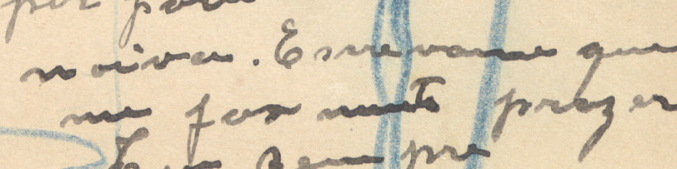

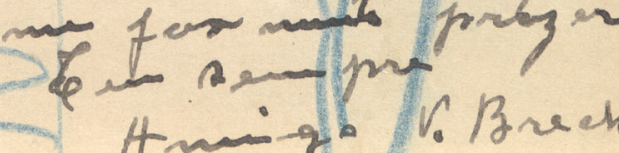




\section{SUMÁRIO}

II Editorial

Dossiê MÁrio de Andrade

I5 A viagem de Mário de Andrade à Amazônia: entre raízes e rotas

ANDRÉ Botelho

SI Mário de Andrade fotógrafo-viajante e a linguagem modernista

Douglas Canjani

83 Engajamento e sacrifício: o pensamento estético de Mário de Andrade

Pedro Fragelli

III Mário de Andrade: O sequestro do desejo e a música popular em versos

Cristiane Rodrigues de Souza

139 Carne para alimento de rádios e discos: o conceito de música popularesca na obra musicológica de Mário de Andrade Juliana Pérez GonzÁlez

I6I Oneyda Alvarenga escreve a Mário de Andrade MARILDA IONTA

I8I Mário de Andrade, Francisco Curt Lange e Carleton Sprague Smith: as discotecas públicas, o conhecimento musical e a política cultural

Flávia Camargo Tonil e Valquíria Maroti Carozze

205 O Pico dos Três Irmãos, obra interrompida de Mário de Andrade: pressupostos de uma edição crítica e genética Marcos Antonio de Moraes

Artigos

233 Imaginário cartográfico na arte contemporânea: sonhar o mapa nos dias de hoje

Gilles Tiberghien 
253 Ritos genéticos editoriais: uma abordagem discursiva da edição de textos

Luciana Salazar Salgado

277 MST conta Boal: do diálogo das Ligas Camponesas com o Teatro de Arena à parceria do Centro do Teatro do Oprimido com o MST

RAFAEL LitVin ViLLAS BôAS

299 Pressentimento da promessa de felicidade: 0 "samba da desilusão" de Paulinho da Viola

Manoel Dourado Bastos

RESENHA

327 retratista e os intelectuais: às voltas com 1964 Anderson Gonçalves, Edu Teruki Otsuka e Ivone Daré

RABELLO

DocumentaÇÃO

35I Trajetória(s) literária(s) de Odette de Barros Mott (19131998): uma narrativa a partir do arquivo pessoal da escritora RaQuel Afonso da Silva

NotícIAs

37I A digitalização de Acervos no IEB-USP: diretrizes para sua reestruturação

375 Homenagem a Elizabeth Travassos 


\section{TABLE OF CONTENTS}

II Editorial

Dossier Mário de Andrade

I5 Mário de Andrade's Journey to the Amazon: Between Roots and Routes

ANDRÉ BotelHo

SI The Travel Photographs of the Brazilian Writer Mário de Andrade and their Connection to the Modernist Visual

Language

Douglas Canjani

83 Engagement and Sacrifice: The Aesthetic Thought of Mário de Andrade

Pedro Fragelli

III Mário de Andrade: The Sequestration of Desire and the Popular Music in Verses

Cristiane Rodrigues de Souza

139 Meat for the Radios and Records: Música Popularesca Concept in Musicological Work of Mário de Andrade

Juliana Pérez González

I6I Oneyda Alvarenga writes to Mário de Andrade MARILDA IONTA

I8I Mário de Andrade, Francisco Curt Lange and Carleton Sprague Smith: Public Disco Collections, Musical Knowledge and Cultural Policy

Flávia Camargo Toni e Valquíria Maroti Carozze

205 O Pico dos Três Irmãos, Mário de Andrade's Interrupted Literary Essay: Presuppositions of a Critical and Genetic Edition

Marcos Antonio de Moraes

\section{Articles}

233 Cartographic Imaginary in Contemporary Art: Dreaming Maps Nowadays

Gilles Tiberghien 
253 Editorial Genetic Rites: A Discursive Approach to Editing Texts Processes

Luciana Salazar Salgado

277 MST Narrates Boal: From the Dialogue of Ligas Camponesas (Peasant Leagues) with the Arena Theatre to the Partnership of Theatre of the Oppressed Center with MST (Landless Movement)

Rafael Litvin Villas Bôas

299 Presentiment of Promesse du Bonheur: The Samba of Disillusion by Paulinho da Viola

Manoel Dourado Bastos

BOOK REVIEW

327 The portraitist and the Intellectuals: Regarding 1964 Anderson Gonçalves, Edu Teruki Otsuka e Ivone Daré RABELLO

Documents

35I Literary Trajectories of Odette de Barros Mott (1913-1998): a narrative on the writer's personal archive RaQuel Afonso da Silva

News

37I Collection's digitization at IEB-USP: guidelines to it's reorganization

375 Tribute to Elizabeth Travassos 


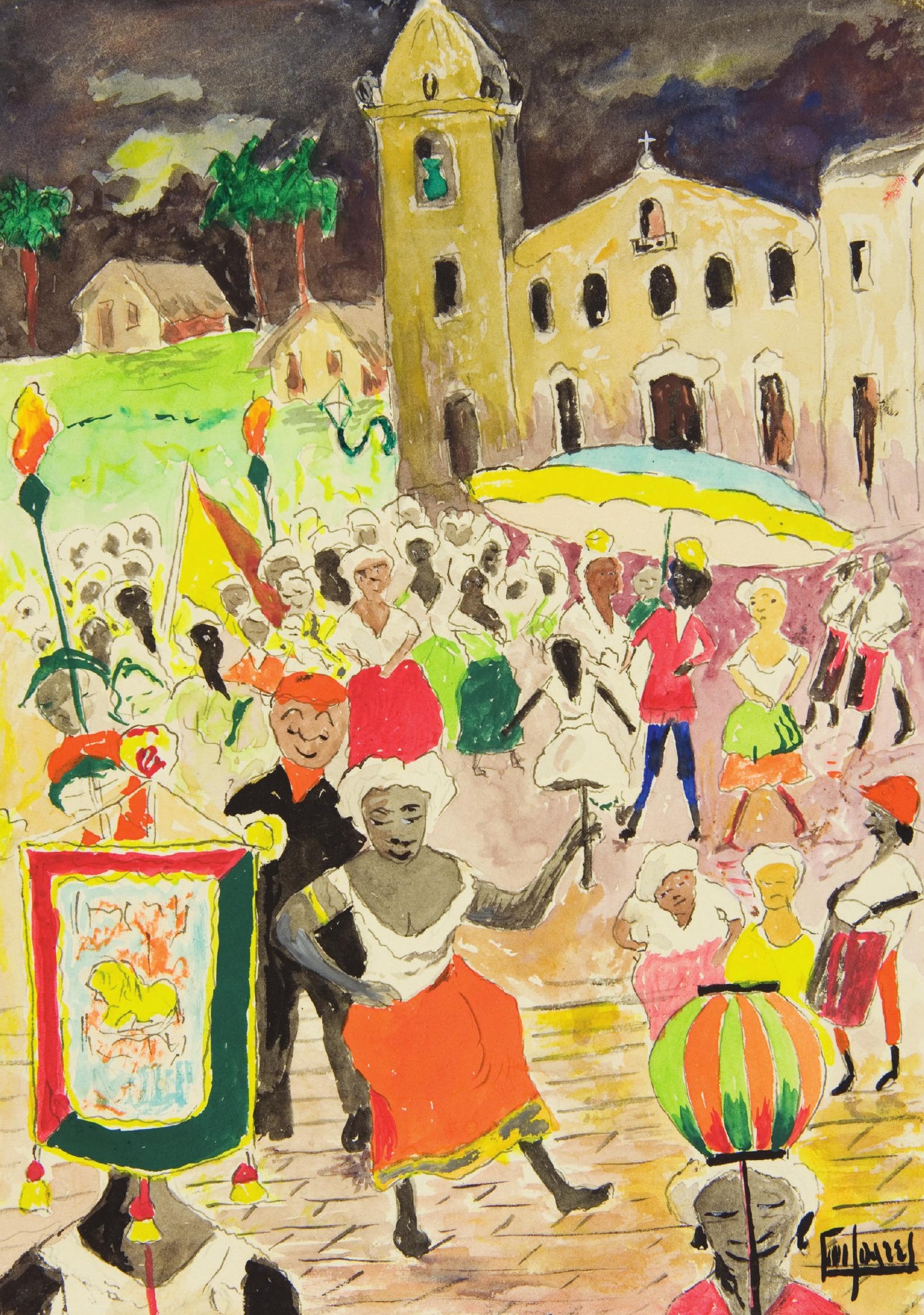




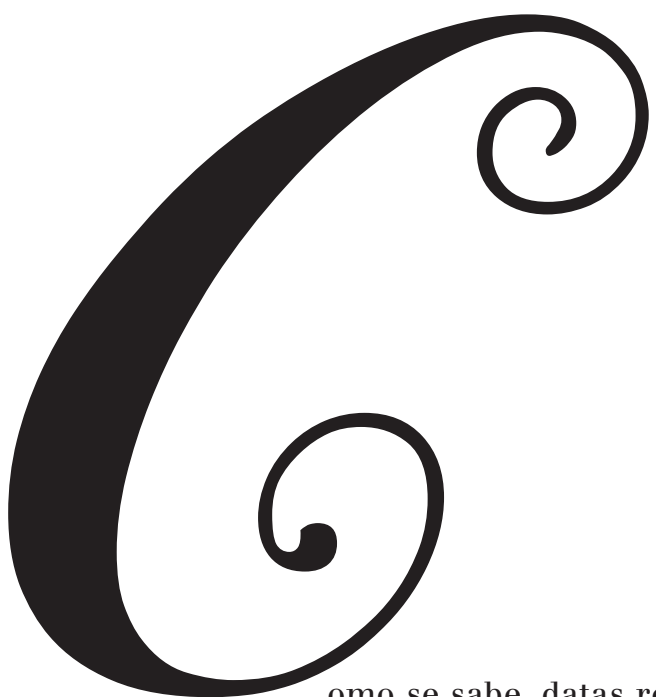

Eu tenho desejo de uma arte que, social sempre, tenha uma liberdade mais estética em que o homem possa criar a sua forma de beleza mais convertido aos seus sentimentos e justiças de tempo da paz. A arte é filha da dor, é filha sempre de algum impedimento vital. Mas o bom, o grande, o livre, o verdadeiro será cantar, as dores fatais, as dores profundas, nascidas exatamente desta grandeza de ser e de viver. Mário de Andrade - Café, terceiro ato, "Dia novo" (São Paulo, 15 de dezembro de 1942.)

omo se sabe, datas redondas sugerem homenagens. Em 2013, são comemorados 110 anos do nascimento de Mário de Andrade. Contudo não seria preciso esperar pela data redonda (e, aliás, um tanto quebrada também) para publicar um dossiê sobre a sua obra. De um lado, a sua trajetória, tão plural quanto complexa, está presente em diversas instâncias da cultura brasileira. De outro, aspectos fundamentais dessa trajetória ainda não foram bem compreendidos. Na junção dos dois lados, os oito artigos do dossiê homenageiam o escritor (e crítico e musicólogo e viajante e fotógrafo e colecionador, entre outros traços biográficos) com pesquisa e com espírito crítico.

No campo das ciências sociais, André Botelho (UFRJ) estuda relatos de Mário de Andrade sobre a viagem que fez à Amazônia em 1927. No campo das artes, fotografias produzidas nessa viagem e na seguinte, ao Nordeste entre 1928 e 1929, são analisadas por Douglas Canjani (PUC-SP). Na imbricação da crítica literária e da filosofia, Pedro Fragelli (Universidade de Paris III) investiga relações entre o pensamento estético marioandradino e o processo histórico brasileiro. A atitude 
interdisciplinar também se faz notar nos dois artigos seguintes: no trabalho de Cristiane Rodrigues de Souza (Centro Universitário Barão de Mauá), cuja análise literária se utiliza de conhecimentos da psicanálise e da musicologia; e no estudo de Juliana Pérez González (USP), radicado na historiografia, mas que examina sentidos diversos da expressão "música popularesca” na obra - musicológica - de Mário de Andrade.

Os três próximos artigos colocam em destaque a pesquisa, dentre vários documentos, da sua correspondência. Dois deles se inscrevem no campo da musicologia. Marilda Ionta (UFV) analisa as cartas trocadas entre Oneyda Alvarenga e Mário. Flávia Camargo Toni (USP) e Valquíria Maroti Carozze (USP) pesquisam as cartas trocadas entre Mário, Francisco Curt Lange e Carleton Sprague Smith, ao lado de outros textos e de dados históricos que esclarecem o diálogo sobre a criação de discotecas públicas. Já Marcos Antonio de Moraes (USP), no campo da literatura, discute pressupostos metodológicos da edição de uma obra planejada e não concluída: um estudo sobre a poesia de Carlos Drummond de Andrade, a de Manuel Bandeira e a de Murilo Mendes.

Na seção de artigos gerais, o campo das artes ocupa o primeiro plano, mas a interdisciplinaridade ainda atua com ênfase. Gilles Tiberghien (Universidade de Paris I), radicado na área da geografia, debate relações entre a arte e a cartografia: "O mapa tem tudo a ver com a arte, pois ele retrata, ele escreve para descrever (literatura) ou permite fazer notações (música) - analogias entre mapas e partituras não faltam”. Rafael Litvin Villas Bôas (UnB) relata "dois momentos do trabalho de Augusto Boal com os movimentos camponeses brasileiros", na década de 1960 e em tempos recentes, analisando experiências nas esferas da cultura e da política. E Manoel Dourado Bastos (UEL), em linha crítica afim com a de Villas Bôas, interpreta "o caráter trágico do samba de Paulinho da Viola". Por sua vez, Luciana Salazar Salgado (UFSCar), no campo da linguística, aborda o trabalho de preparação de textos autorais no mercado editorial.

Em Resenha, Anderson Gonçalves, Edu Teruki Otsuka e Ivone Daré Rabello discutem Martinha versus Lucrécia: ensaios e entrevistas, de Roberto Schwarz. Em Documentação, Raquel Afonso da Silva apresenta a obra de Odette de Barros a partir do arquivo pessoal da escritora. Notícias publica texto de José Hermes Martins Pereira sobre a Seção de Digitalização do IEB; e traz a homenagem da área de música do Instituto a Elizabeth Travassos.

Walter Garcia

Editor Responsável 